\title{
Présentation du répertoire Autografi dei letterati italiani
}

\section{Matteo Motolese et Emilio Russo}

\section{(2) OpenEdition}

9 Journals

Édition électronique

URL : https://journals.openedition.org/genesis/4809

DOI : 10.4000/genesis.4809

ISSN : 2268-1590

Éditeur :

Presses universitaires de Paris Sorbonne (PUPS), Société internationale de génétique artistique littéraire et scientifique (SIGALES)

Édition imprimée

Date de publication : 15 décembre 2019

Pagination : 157-164

ISBN : 979-10-231-0650-3

ISSN : $1167-5101$

\section{Référence électronique}

Matteo Motolese et Emilio Russo, "Présentation du répertoire Autografi dei letterati italiani », Genesis [En ligne], 49 | 2019, mis en ligne le 01 décembre 2020, consulté le 03 septembre 2021. URL : http:// journals.openedition.org/genesis/4809; DOI : https://doi.org/10.4000/genesis.4809 


\title{
Présentation du répertoire Autografi dei letterati italiani*
}

\author{
Matteo Motolese et Emilio Russo
}

\section{Le projet}

L'idée première d'un recensement des manuscrits autographes des hommes de lettres italiens est née vers 2003. Au cours de nos travaux de doctorat sur la littérature et la langue de la Renaissance, nous avions été confrontés à la difficulté d'identifier et d'attribuer non seulement certains manuscrits d'auteur mais également des textes imprimés comportant des annotations : l'absence d'outils permettant de s'orienter dans ce panorama complexe nous a conduits à imaginer un répertoire dont la finalité serait d'organiser l'ensemble des connaissances à disposition, tout en ouvrant de nouvelles pistes de recherche.

La mise en œuvre du projet s'est faite progressivement, après diverses tentatives. L'idée initiale était de cataloguer les livres annotés de la Bibliothèque Corsiniana et de l'Académie des Lincei, une sorte de projet pilote portant sur un corpus restreint et qui devait définir l'orientation et les modalités des travaux à mener. Le projet put s'appuyer également sur les bibliographies spécialisées fournies par les chercheurs qui ont donné ces dernières décennies, à l'occasion de colloques ou de publications de catalogues de bibliothèques ${ }^{1}$, un nouvel essor à l'étude des textes annotés et des autographes. Citons en premier lieu les recherches menées par Armando Petrucci, depuis son ouvrage sur les écrits de Pétrarque jusqu'au panorama qu'il consacre aux textes autographes dans la Letteratura italiana Einaudi ${ }^{2}$; mentionnons aussi le projet de cartographie des manuscrits italiens de la Renaissance dirigé pendant plus de trente ans par Paul Oskar Kristeller avec l'aide d'un important réseau de collègues et collaborateurs, et qui portait une attention particulière aux textes autographes de la tradition ${ }^{3}$; signalons enfin, l'anthologie coordonnée par Albinia de la Mare, The Handwriting of Italian Humanists, où la description des manuscrits associée à leur reproduction partielle s'offre comme un modèle du point de vue éditorial ${ }^{4}$.
Ces travaux montraient qu'il était possible d'unir une analyse détaillée de l'atelier d'écriture de chaque auteur (ses manuscrits, ses livres annotés) avec un recensement à large échelle des autographes. Notre idée était donc d'établir une base documentaire stable et organisée, qui regroupe les très nombreuses données bibliographiques disponibles sur les papiers des plus grands auteurs de notre tradition littéraire, en les accompagnant de reproductions de documents. La constitution de ce répertoire n'était cependant pas une fin en soi : elle entendait être le point de départ d'une réflexion sur les dynamiques complexes de production et de circulation des documents originaux des premiers siècles de la littérature italienne.

(*) Une version en anglais de cette chronique (M. Motolese, E. Russo, "The "Autographs of the literati Italian" Project») est parue, avec de légères différences, dans Ecdotica, $\mathrm{n}^{\circ}$ 11, 2014, p. 205-215.

1. Mentionnons V. Fera, G. Ferraù, S. Rizzo (dir.), Talking to the Text: Marginalia from Papyri to Print, Messine, Centro interdipartimentale di studi umanistici, 2002; E. Barbieri (dir.), Nel mondo delle postille. I libri a stampa con note manoscritte : una raccolta di studi, Milan, CUSL, 2002; E. Barbieri, G. Frasso, Libri a stampa postillati, Milan, CUSL, 2003. Rappelons aussi, pour élargir à l'international : R.C. Alston, Books with Manuscript: a short Title Catalogue of Books with Manuscript Notes in the British Library, Londres, The British library, 1994.

2. A. Petrucci, La scrittura di Francesco Petrarca, Cité du Vatican, Bibliothèque Apostolique Vaticane, 1967; Id., «La scrittura del testo », dans A. Asor Rosa (dir.), Letteratura italiana, vol. IV. L'interpretazione, Turin, Einaudi, 1985, p. 283-310 (avec 40 planches).

3. P. O. Kristeller (dir.), Iter Italicum. A Finding List of Uncatalogued or Incompletely Catalogued Humanistic Manuscripts of the Renaissance in Italian and other Libraries, Londres-Leyde, The Warburg Institute-Brill, 1963-1997, 6 vol.

4. A. De La Mare, The Handwriting of Italian Humanists, Oxford, Association internationale de bibliophilie, 1973 (le vol. I concernait Pétrarque, Boccace, Coluccio Salutati, Niccolò Niccoli, Le Poge, Bartolomeo Aragazzi di Montepulciano, Sozomeno di Pistoia, Giorgio Antonio Vespucci). Voir aussi A. De La Mare, L. Nuvoloni, Bartolomeo Sanvito. The Life \& Work of a Renaissance Scribe, éd. A. Hobson et C. de Hamel, Paris, Association internationale de bibliophilie, 2009. 
Cette entreprise, certes fondée sur une idée simple, s'avérait toutefois difficile à mettre en œuvre pour des jeunes chercheurs ne disposant pas de structures institutionnelles en mesure de les épauler. D'autres projets de recherche similaires s'étaient heurtés, quelques années auparavant, à d'énormes obstacles, en matière de repérage et de vérification des données, de consultation directe des sources, voire d'accès même à l'information. Toutefois, notre fragilité était un atout : elle favorisa la mise en place d'une pratique largement collaborative du travail, libérée de toute contrainte administrative et des engagements liés aux financements. Les discussions avec nos amis et maîtres les plus proches ${ }^{5}$, l'accueil enthousiaste réservé par de nombreux chercheurs à notre hypothèse de travail, nous persuadèrent de deux choses : de la nécessité de cet outil qui allait permettre de s'orienter dans le monde manuscrit des premiers siècles de notre littérature et favoriserait un nouveau progrès des connaissances en ce domaine, après l'entreprise pionnière de Kristeller; plus généralement, de la force et de la valeur d'un versant des études philologiques capable de porter, à une époque où ce domaine de recherche semblait affaibli, un regard renouvelé sur les auteurs à travers le prisme spécifique de l'autographie. Le soutien de Paolo Procaccioli fut dès lors déterminant : c'est à lui que l'on doit notamment l'idée d'organiser à Forlì en 2008 un colloque destiné à lancer officiellement ce chantier de recherche sur les autographes, en commençant par une vaste réflexion sur le rapport entre autographie et littérature italienne 6.

\section{Papier ou numérique?}

L'une des premières questions qu'il nous fallut affronter était celle de la forme à donner au répertoire : papier ou numérique. Nous avons opté pour une double solution : d'abord une série de volumes imprimés, qui constitueraient le noyau dur de l'entreprise, puis une conversion numérique, sujette à des corrections, des améliorations, des enrichissements, mais fondée principalement sur les données réunies pour la base documentaire imprimée. Ce choix fut dicté par des raisons tout à la fois pratiques et scientifiques. Du point de vue scientifique, nous étions convaincus qu'une publication papier restait la condition sine qua non d'une diffusion satisfaisante de notre recherche dans le champ des humanités en Italie, non pas tant pour toucher des chercheurs peu enclins à naviguer dans un environnement numérique, mais pour convier le public à une lecture attentive des fiches et des outils iconographiques proposés (notre but était aussi de stimuler la mémoire photographique des lecteurs et de leur suggérer, le cas échéant, de nouvelles pistes pour l'identification des documents autographes). Quant aux raisons pratiques, il y en avait plusieurs : une publication papier jouissant en Italie d'une meilleure reconnaissance dans le domaine scientifique, ce choix nous permit d'obtenir l'adhésion volontaire et désintéressée de nombreux collègues; par ailleurs, en l'absence d'un financement adapté, le coût pour la conception et la maintenance d'un portail numérique s'avérait trop élevé.

\section{Bornes chronologiques}

Nous avons fait deux choix importants en concevant le répertoire. Le premier fut de limiter le recensement aux premiers siècles de la littérature italienne, en partant des origines pour arriver à la fin du Xvie siècle. Nous nous sommes appuyés, pour définir ces bornes, sur l'exemple de l'Iter Italicum de Kristeller, mais aussi sur la conviction qu'un projet de ce type était surtout utile pour les premiers siècles de notre littérature dans la mesure où l'étude des documents autographes, qui permet de saisir les dynamiques et la circulation de la culture lettrée, est indispensable à l'analyse des textes de cette période. Notre second choix fut de nous fonder, pour la constitution du répertoire, non pas sur des critères chronologiques, alphabétiques ou topographiques, mais sur l'état des recherches en cours, en ne publiant que les travaux et recensements jugés suffisamment mûrs et fiables. L'organisation du répertoire prit ensuite la forme de tomes

5. Les discussions initiales avec Corrado Bologna, Giuseppe Frasso et Armando Petrucci furent particulièrement précieuses. Nous leur sommes reconnaissants pour leur confiance, leurs conseils et leurs suggestions. 6. Le colloque, organisé par le Centre Pio Rajna, la Biblioteca Comunale Saffi et la Fondazione Garzanti de Forlì, s'est tenu les 24-27 novembre 2008 : le projet fut alors officiellement présenté à la communauté scientifique internationale et nous avons pu, grâce à la participation de nombreux spécialistes, recueillir les ultimes indications théoriques et pratiques pour la réalisation de l'ouvrage. Le premier volume des Autografi dei letterati italiani, comportant une trentaine de fiches d'auteurs du XvI ${ }^{\mathrm{e}}$ siècle, parut quelques mois plus tard, suivi par les actes du colloque : G. Baldassarri, M. Motolese, P. Procaccioli, E. Russo (dir.), Di mano propria : gli autografi dei letterati italiani, Rome, Salerno, 2010. 
chronologiques (Le Origini e il Trecento, Il Quattrocento, Il Cinquecento), dotés d'un index conclusif qui devait en garantir une utilisation fonctionnelle.

Définir le degré d'exhaustivité que devait avoir le répertoire, à l'intérieur des bornes chronologiques ainsi fixées, fut une étape importante. Nous avons choisi de ne pas nous en tenir aux seuls écrivains de premier rang, aux auteurs des nombreux chefs-d'œuvre qui scandent l'histoire des premiers siècles de notre littérature; nous avons décidé d'y inclure aussi certaines figures de moindre stature afin de reconstituer le tissu compact de la société des hommes de lettres. Ont donc été inclus les auteurs d'œuvres jugées significatives, les protagonistes des réseaux de circulation lettrée ou d'une réflexion théorique sur les statuts et les genres littéraires, les anciens commentateurs des classiques, les traducteurs, les premiers représentants d'une approche philologique, etc., notamment pour le premier volume consacré aux hommes de lettres des origines et du XIve siècle. Par ailleurs, outre les œuvres littéraires, nous avons inclus les écrits documentaires (actes notariaux, accords commerciaux, lettres privées, mémoires de voyage, etc.) dans l'objectif d'offrir un très large éventail de documents autographes. C'est la raison pour laquelle nous avons choisi de recourir, y compris dans le titre de notre ouvrage, au terme de «letterati», dont l'acception large donne une idée de l'ouverture d'esprit de notre entreprise et des recherches qu'elle entend susciter.

\section{Modèle de fiches pour les dossiers d'auteur}

Pour commencer, il nous a fallu déterminer un modèle de dossier d'auteur. Pour ce faire, nous avons rédigé deux exemples de fiches relatives aux autographes de deux auteurs très différents (Castelvetro et Marino), chacun représentatif des problèmes et des questions que soulevait la constitution du répertoire. L'abondance prévisible des documents à recenser nous a conduits à choisir un modèle de fiche extrêmement synthétique pour chaque manuscrit ou livre annoté. La présentation se structure en trois parties :

1. une première section mentionne la ville et la bibliothèque où est conservé l'autographe ou le livre annoté, ainsi que la cote des documents et l'éventuelle numérotation des feuillets;
2. une deuxième section comporte une description succincte du contenu : les titres des œuvres, les destinataires des lettres, ainsi que les incipit des compositions poétiques;

3. une troisième section présente une bibliographie spécifique pour chaque manuscrit ou livre annoté, destinée à fournir au lecteur des indications succinctes pour s'orienter parmi les études disponibles.

Voici un exemple précis de fiche sur une lettre de l'Arétin, publiée dans le volume paru en 2009:

Mantova, ASMn, Autografi 8 3, cc. 20r-20bisv. • Lettera con sonetto (Qui iace Adrian sesto, homo divino) al marchese Federico II Gonzaga (Milano, s.d.; Larivaille propone di datarla a fine novembre 1523). • Luzio 1890: 690-92; Cesareo 1901 : 177 n. 3, 183 n. 8, 190; Pasquinate 1983 : 328-29, 976; Larivaille 1989 : 137; Kristeller : VI 21.

C'est à partir de ce modèle de présentation qu'ont pris forme les fiches consacrées à chaque auteur, structurées elles aussi en plusieurs sections :

1. une section introductive, visant à reconstituer le destin des papiers de l'homme de lettres et à indiquer les recherches qu'il faut encore mener;

2. le recensement des autographes et des livres annotés selon le modèle présenté ci-dessus ${ }^{7}$;

3. un commentaire paléographique sur les particularités des différents styles d'écriture manuscrite de l'auteur, qui puisse servir de base à de nouvelles attributions :

4. une section conclusive comportant des planches représentatives de la façon d'écrire de chaque auteur. Conçue pour les volumes imprimés, cette section ne propose qu'un nombre limité de reproductions (4 à 6 , en général), mais propres à illustrer diverses époques et divers contextes d'écriture : notes préparatoires et mises au net, lettres et compositions en vers, annotations de manuscrits ou de livres imprimés, etc.

7. Nous avons adopté cette distinction entre «autographes» et «livres annotés » par simplicité, même si elle soulève des objections prévisibles en matière de terminologie. En effet, non seulement les annotations dans les livres sont autographes, mais il n'est pas toujours possible de distinguer clairement les interventions d'un auteur sur ses propres textes et ses notes de lecture (c'est la raison pour laquelle, dans certains cas, l'item apparaît dans les deux sections). Pour des raisons de clarté, nous avons également inclus dans cette section des documents hybrides, mais dont la mention était utile, tels que les livres portant une dédicace de Giordano Bruno. 
Nous avons ensuite soumis ce modèle de fiche à un groupe de chercheurs qui confirmèrent la validité de notre hypothèse de travail et suggérèrent un certain nombre d'améliorations possibles : par exemple, mieux mettre en relation les commentaires sur l'écriture manuscrite et les planches illustratives, afin que le lecteur puisse saisir immédiatement les spécificités de chaque écriture; ou encore faire mention des manuscrits dont le caractère autographe est réfuté, ou mentionner les manuscrits cités dans d'anciennes bibliographies et dont la trace est aujourd'hui perdue, afin de permettre d'éventuels recouvrements. Nous avons prévu d'adjoindre aux sections des autographes et des livres annotés des sections parallèles consacrées aux documents dont l'attribution reste incertaine.

\section{De la fiche au répertoire}

Une fois défini dans ses grandes lignes, le modèle de fiche allait connaître par la suite quelques ajustements pour s'adapter au cas des manuscrits les plus anciens 8 ; le projet est entré alors dans une phase de développement plus dynamique, avec la collaboration de nombreux autres chercheurs et le choix des responsables des différentes sections chronologiques.

Les quatre premiers volumes, fruits d'un travail mené en parallèle et d'un dialogue constant entre équipes de chercheurs, ont paru entre 2009 et 2013 : Le Origini e il Trecento vol. I, Il Quattrocento vol. I, et Il Cinquecento vol. I et II 9 . La moitié du chemin est parcourue : d'ici 2021, la parution du vol. II du tome Le Origini e il Trecento, celle du vol. II de Il Quattrocento et du vol. III de Il Cinquecento compléteront l'ouvrage; paraitra un huitième et dernier volume contenant un l'index général. L'ensemble aura permis de recenser les manuscrits autographes et les livres annotés d'environ deux cents écrivains actifs en Italie entre le XIII et le XVIe siècle.

Difficile cependant de donner une estimation du nombre de documents qui auront été décrits, une fois le projet achevé. Le catalogue devrait contenir, d'après les données dont nous disposons, plusieurs milliers d'éléments, tant pour la section des manuscrits autographes que pour celle des livres annotés : à ce jour, 2771 éléments ont été catalogués dans la section des autographes (plus 37 cas d'attribution douteuse) et 1222 dans la section des livres annotés (dont 37 cas douteux également) pour un total de 113 auteurs (tableau 1).
Ces milliers de fiches sont l'œuvre de quatre-vingt-quinze chercheurs qui ont gracieusement partagé leurs travaux et mené des vérifications dans les bibliothèques afin d'esquisser un cadre général dans lequel réorganiser les savoirs déjà constitués et dégager de nouvelles pistes de recherche. Cette enquête de fond nous a naturellement conduits à retrouver et identifier de nouveaux documents, mais aussi à remettre en question des attributions antérieures. Elle a également permis de réviser la datation de manuscrits ou de repenser les relations entre certains d'entre eux. Pensons par exemple au réexamen complet des autographes et de la bibliothèque de Boccace, que l'on doit à Maurizio Fiorilla et à Marco Cursi, qui fut à l'origine de découvertes et de rectifications dans la datation de plusieurs documents; ou aux nouvelles attributions relatives à la bibliothèque de Cristoforo Landino, que l'on doit à Valerio Sanzotta et Teresa De Robertis, ou encore à la reconstitution du laboratoire philologique d'Angelo Poliziano, essentiel pour l'Humanisme italien, qui revient au mérite d'Alessandro Daneloni; citons, pour le volume consacré au XVIe siècle, les informations réunies sur Pietro Bembo (fig. 1) et Sperone Speroni, sur Lodovico Castelvetro et Giorgio Vasari, mais aussi sur de nombreux «polygraphes» de la Renaissance (l'Arétin, Ruscelli, Dolce, Doni, etc.), sur l'activité desquels les travaux de Paolo Procaccioli, Paolo Marini et Carlo Alberto Girotto ont jeté une lumière nouvelle.

Notre souhait, toutefois, est que l'ensemble des informations et des reproductions réunies dans ces volumes favorisent l'essor d'études nouvelles et approfondies sur les manuscrits. La masse des données ici présentées et la possibilité de les recouper entre elles grâce aux index (en particulier l'index du dernier volume) doit servir de fondement à une réflexion méthodologique générale sur la pratique de l'annotation, sur les modes de partage et de circulation des textes et sur la langue des auteurs des premiers siècles de la littéraire italienne.

8. Voir les précisions dans la section «Avvertenze» du volume Le Origini e il Trecento, éd. G. Brunetti, M. Fiorilla, M. Petoletti, Rome, Salerno, 2013, p. XIII

9. Le Origini e il Trecento, op. cit.; F. Bausi, M. Campanelli, S. Gentile, J. Hankins (dir.), Il Quattrocento, I, Rome, Salerno, 2014; M. Motolese, P. Procaccioli, E. Russo (dir.), Il Cinquecento, I et II, Rome, Salerno, 2009 et 2014 . 
Tableau 1 : Le contenu du catalogue*

\begin{tabular}{|l|c|c|c|}
\cline { 2 - 5 } \multicolumn{1}{c|}{} & Hommes de lettres & Autographes & \multicolumn{2}{c|}{ Livres annotés } \\
\hline Le Origini e il Trecento I & 26 & $156[+2]$ & $50[+2]$ \\
\hline Il Quattrocento I & 26 & $706[+24]$ & $424[+18]$ \\
\hline Il Cinquecento I & 30 & $1230[+10]$ & $542[+6]$ \\
\hline Il Cinquecento II & 31 & $2771[+37]$ & $1222[+37]$ \\
\hline Total & 113 & {$[+11]$} \\
\hline
\end{tabular}

(*) Entre crochets figurent les cas d'attribution douteuse. Sont comptabilisés pour les autographes et les livres annotés les éléments catalogués, ou entrées, des diverses sections des volumes. Chaque élément catalogué correspond à une cote de la bibliothèque ou du fonds d'archives où le document est conservé.

\section{De l'imprimé à Internet}

La version numérique du projet valorise l'ensemble de ces travaux et les rend plus facilement accessibles. La réflexion sur le développement de ce versant du projet a débuté dès 2011 grâce à un financement destiné aux chercheurs de moins de 40 ans attribué par le ministère italien de l'Enseignement supérieur et de la Recherche10. La mise en ligne du contenu, après un lent travail de structuration, a été effectuée à l'automne 2016. Désormais, l'ensemble des documents peut être librement consulté sur le site www. autografi.net. Comme pour les volumes imprimés, le développement du portail a été le fruit d'un travail d'équipe qui a impliqué un grand nombre de collègues, sous la coordination des responsables des trois institutions hôtes ${ }^{11}$.

La conception et la réalisation du portail Autografi dei letterati italiani avaient deux objectifs :

a) permettre la consultation de l'intégralité du répertoire avec une augmentation significative du nombre de documents numérisés;

b) favoriser les recherches transversales par le biais d'une agrégation souple des données disponibles.

En matière de reproductions, un accord passé avec le Ministère italien de la culture (MiBAC) dès le mois de juin 2013, nous a permis de lancer une campagne de reprographie dans les bibliothèques d'État. Quarante mille documents manuscrits ont ainsi été numérisés. D'autres accords avec des bibliothèques et archives italiennes ou étrangères sont en projet (avec la Biblioteca Comunale Ariostea de Ferrare, pour les manuscrits de l'Arioste et du Tasse, par exemple), qui nous permettront d'accroître considérablement le nombre de documents reproduits sur le site. Notre ambition est de fournir, d'ici quelques années, une reproduction numérique partielle ou intégrale de tous les documents recensés. Nous comptons, pour ce faire, sur la coopération des institutions propriétaires des manuscrits originaux et sur un abaissement des droits de reproduction; ou encore sur l'accès partagé aux campagnes de numérisations déjà effectuées par certaines bibliothèques (comme c'est déjà le cas avec la Bibliothèque Apostolique Vaticane et la BnF).

$S$ 'il offre de nombreux avantages pour interroger une base de données et réagencer librement les éléments de cette base, le numérique présente cependant le risque de rendre moins claire la perception de l'ensemble. C'est la raison pour laquelle une section dédiée du portail permet d'accéder à des «parcours » de consultation, dont le but est d'offrir une vision panoramique de certains contenus du répertoire, à travers l'agrégation de documents appartenant à des

10. Il s'agit d'un financement FIRB 2010 - Futuro in ricerca qui réunit l'Università di Roma «La Sapienza (Matteo Motolese, coordinateur national), l'Università Cattolica del Sacro Cuore de Milan (resp. : Marco Petoletti) et l'Università di Roma 3 (resp. : Maurizio Fiorilla). Avant cela, le projet avait obtenu un financement PRIN 2008, avec la participation de cinq universités : Università della Tuscia (Paolo Procaccioli, coordinateur national), Università di Perugia (resp. : Antonio Ciaralli), Università di Roma «La Sapienza» (resp. : Matteo Motolese), Università Cattolica del Sacro Cuore de Milan (resp. : Marco Petoletti), Università di Roma 3 (resp. : Maurizio Fiorilla).

11. Auxquels s'ajoute Francesca Ferrario pour le domaine informatique. Pour un tableau d'ensemble des responsables et collaborateurs, voir : http:// www.autografi.net/it/staff/. 


\section{NOTA SULLA SCRITTURA}

In apparente contrasto con la mirabile operosità di letterato, poeta e storiografo, nonostante un'educazione attenta agli studi umanistici di greco e di latino e a dispetto di un'attività svolta in campo editoriale di altissimo profilo e soprattutto innovativa sul piano ortografico, la grafia di P. B. mostra connotati di asciutta essenzialità. È, in effetti, una minuta corsiva di matrice umanistica del tutto ripulita da orpelli, scarna ed essenziale: si direbbe che sia la mano di persona interessata piú ai contenuti e molto meno ai caratteri estrinseci della loro comunicazione. Inclinata con moderazione a destra, di modulo ridotto, aguzza e ricca di legature: sempre realizzate per quei segni alfabetici in possesso di un tratto in uscita ascendente da sinistra verso destra $(i, l, m, n, t$ nella versione semplificata in un tempo solo), ovvero orizzontale ( $c$ e, $t$ col suo secondo tratto), esse coinvolgono spesso piú lettere e talvolta anche lettere bisognose, per legare, di una pronunciata linea di congiunzione (cosi la $a$ in Tebaldo, 2 r. 3; mentre rimane accennata, ma non attuata, quella di $e h$ in ehora, 2 r. 2). Tra i connotati peculiari si può segnalare la $a$ il cui occhiello è talmente stretto da risultare praticamente non visibile (ma che sia presente lo testimonia la piccola testa di attacco a sinistra); la $o$ che resta aperta quando in legamento anteriore (torto, come 2 r. 6; suo, 2 r. 7); il legamento sp, la $d$, sempre con asta obliqua, in un tempo solo a partire dall'occhiello destinato a rimanere aperto e acuto; la $z$ eseguita in un unico colpo di penna sinuoso di grande dimensione sia sopra, sia sotto il rigo (innanzi, 1 ultima riga della colonna centrale; marzo, 2 nella datatio); il compendio per $q($ ue) col segno abbreviativo basso sull'asta della $q$. Da notare l'uso di una interpunzione che si avvale, com'era consuetudine del tempo, della virgola per la pausa breve, del punto e virgola e del doppio punto per segnalare una pausa di media intensità, del punto seguito da maiuscola per quella prolungata. Con l'accento, infine, è precisata qualche forma verbale. Una scrittura, quella del B., povera di manifeste evoluzioni nel corso degli anni e spogliata pure di quei segni diacritici da lui invece, con audacia non priva di contestazioni, introdotti nella stampa. Una scrittura, nella sostanza, non troppo dissimile da quella del padre Bernardo (si vede in $M A P,{ }^{1}$ tav. Lxiv), ma, rispetto a questa, di gran lunga meno ariosa ed elegante. Per quanto riguarda la produzione di testi in lingua greca è opportuno richiamare qui la descrizione di Eleuteri e Canart: essi sono vergati in una «scrittura minuta e corsiveggiante in cui sono da rilevare il beta a occhielli, il lambda con il secondo tratto curvilineo che termina sotto il rigo di base, il chi di forma ampia ${ }^{2}{ }^{2}$ Pure da menzionare sono l'eta $\mathrm{e}$ il theta prevalentemente maiuscoli e la struttura tondeggiante del phi. ${ }^{3}$ Eleuteri $\mathrm{e}$ Canart inseriscono la grafia del B., sebbene questa lo rappresenti solo « in una certa misura (op. cit., p. 12), nel filone che gli studiosi chiamano inclinato corsivo, una «tendenza generale», con scritture non eccessivamente ricche di legamenti, dotato di discreta fortuna nel sec. XVI. [A. C.]

\section{RIPRODUZIONI}

1. Città del Vaticano, BAV, Vat. Lat. 3210, c. 157r. Autografo delle Prose della volgar lingua (III 63), precedente la princeps del 1525. La nitidezza delle giunte, i segni di richiamo, l'impaginazione dei versi (con iniziale non allineata e su diversa riga) e le citazioni in maiuscoletto depongono a favore di uno scaltro gusto tipografico. La mezzaluna in calce, rinvio a una giunta a c. $156 v$, compare anche nell'Igino ambrosiano di tav. 6 a.

1. Autografi dell'Archivio Mediceo avanti il principato, a cura di M. Fortuna e C. Lunghetti, Firenze, Corradino Mori, 1977. 2. P. Eleuteri-P. Canart, Scrittura greca nell'Umanesimo italiano, Milano, Il Polifilo, 1991, p. 95. 3. S. Bernardinello, Autografi greci e greco-latini in Ocidente, Padova, Cedam, 1979, pp. 60-61.

2. Forli, BCo, Raccolte Piancastelli, Sez. Autografi secc. XII-XVIII, 7, num. 5, Bembo Pietro. Lettera autografa a Angelo Colocci, Venezia, 9 marzo 1539.

3. Wien, ÖN, Vind. Pal. 10245.1, c. 7or. Rime, con correzioni autografe (qui le ottave 31-33 delle Stanze). Ricordato in una lettera a Girolamo Quirini dell'11 novembre 1541 (Вемво 1987-1993: num. 2297), il codice contiene correzioni che arrivano al 1545 .

4. Paris, BnF, Rés. Yd 208, c. 65v. Convivio di Dante Alighieri Fiorentino (colophon: «Impresso in Firenze per ser Francesco bonaccorsi $\mathrm{Nel}$ anno mille quattrocento novanta Adi xx. Di septembre»). Lincunabolo reca note anche di Bernardo Bembo. Qui, un esempio dei notabilia apposti da Pietro Bembo.

5. Città del Vaticano, BAV, Barb. Gr. 280, c. 1r. Lettera greca a Demetrio Mosco, Messina, $1^{\circ}$ gennaio 1493.

6a. Milano, BAm, M 12 sup., vol. In p. 210. In calce: "volve ad hoc signum» seguito da una mezzaluna rovesciata. Questa, come altre note autografe di Bembo presenti nel codice, ricostruiscono la fascicolazione del De syderibus tachigrafico di Igino, vergato su un palinsesto del sec. IX. Inviato nel febbraio del 1513 a Bembo da Giulio II, perché lo interpretasse, Bembo ringrazia il pontefice con lettera del 21 febbraio di quell'anno (Вемво 1987-1993: num. 326).

6b. Windsor, Eton College Library, 124, c. 138r. Iohannis Diaconi Vitae S. Gregorii Libri IV, secc. XI-XII. Nota di Pietro Bembo in due tempi diversi: "vehementer existimo ut verba celestis oraculi restringa(m) sub regulis Donati l Fol. 125. a *. E "t $(\mathrm{em}) \mathrm{p}(\mathrm{o})$ re Adriani $\mathrm{p}(\mathrm{a}) \mathrm{p}(\mathrm{ae})$ fol. 80. a».

6c. Windsor, Eton College Library, 135, c. $3 v$. Seneca, Epistulae ad Lualium, sec. XII. Appartenuto a Bernardo Bembo fin dagli anni '8o del Quattrocento, il codice reca anche note di Pietro (qui: «Ad hanc respondet Seneca in ea epistola, Ave mi Pauli. Nisi mihi nominique meon).

Fig. 1 : M. Motolese, P. Procaccioli, E. Russo (dir.), Autografi dei letterati italiani. Il Cinquecento, t. I, Rome, Salerno, 2009, montage des p. 58-59 (gauche) et p. 60 (droite). 


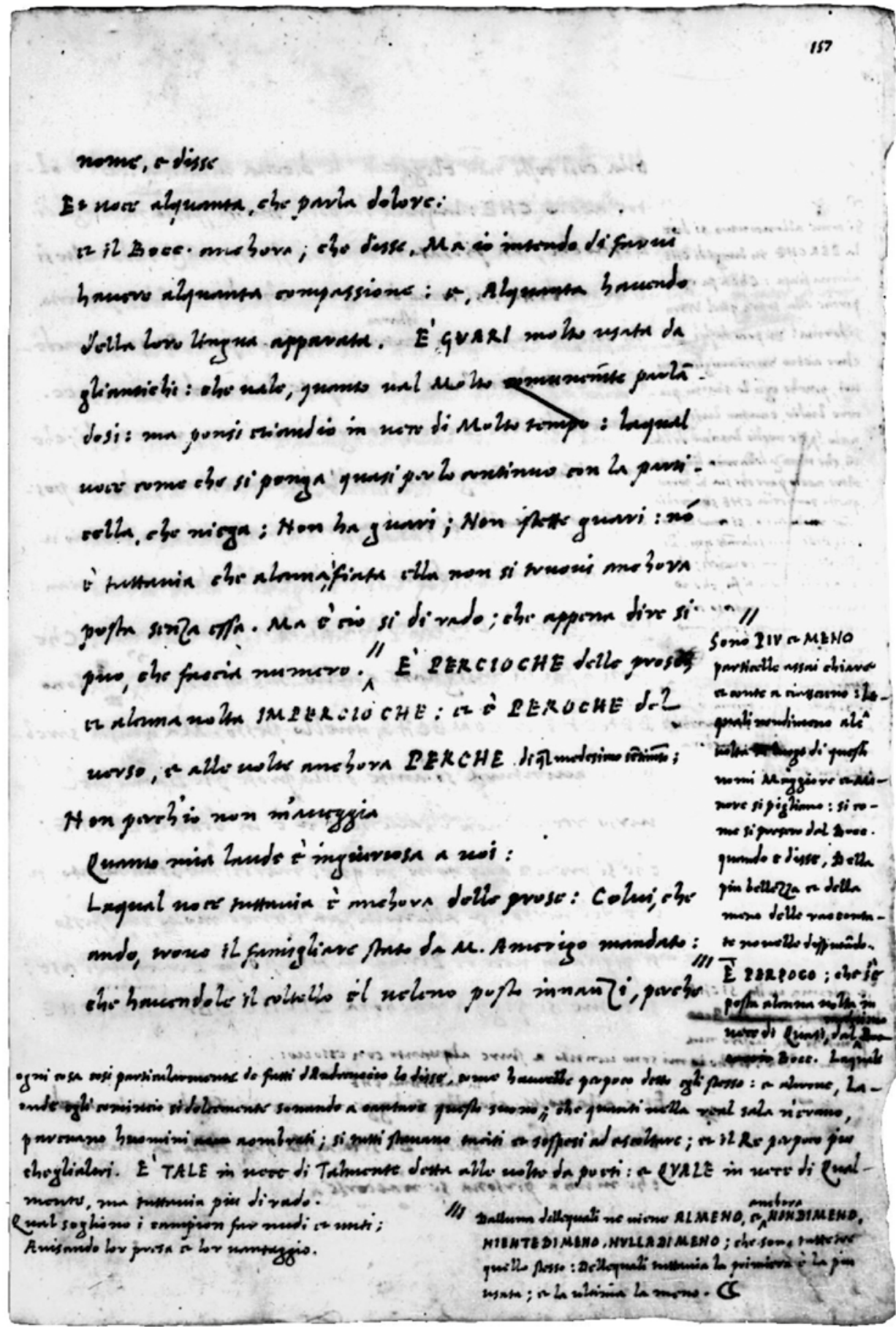

1. Città del Vaticano, BAV, Vat. Lat. 3210, c. 157 r.

Ci-dessus figure un manuscrit autographe des Prose della volgar lingua de Pietro Bembo (III, 63), qui précède l'édition princeps de 1525 (Cité du Vatican, Bibliothèque Apostolique Vaticane, Vat. Lat. 3210, f. 157 r). Å gauche se trouvent l'analyse paléographique et la description des huit documents originaux de Bembo présentés dans le volume. La «Nota sulla scrittura» explique : «En opposition apparente avec l'exceptionnelle productivité de Bembo, à la fois lettré, poète et historiographe [...] et malgré sa remarquable activité d'éditeur, source d'innovations notamment sur le plan orthographique, la graphie de l'auteur est sobre et va à l'essentiel [...] : on dirait la main d'une personne plus intéressée par les contenus que par les caractéristiques extrinsèques de la communication [...]. Une écriture dépourvue d'évolutions manifestes au fil des ans et dépouillée également des signes diacritiques que Bembo lui-même avait, avec une audace qui ne manqua pas de soulever des protestations, introduits dans l'imprimerie.» 
typologies ou à des catégories déterminées, que ce soit pour les autographes (par exemple : matériels préparatoires, copie d'une ouvre personnelle, copie de l'œuvre d'un tiers...) ou pour les livres annotés (classiques, patristiques...). Pour cette même raison, nous avons choisi de consacrer une section spécifique aux lettres (avec un filtre de recherche dédié), afin de mettre en valeur une typologie d'autographes particulièrement vaste du point de vue quantitatif et fondamentale pour comprendre comment se constituent à chaque époque les réseaux culturels. Par la suite, notre intention est de poursuivre le développement de la base de données, en commençant par l'aspect linguistique : il s'agira de proposer la transcription de l'ensemble des documents numérisés, afin qu'à l'avenir tous puissent être interrogés par le moteur de recherche (mais il est encore trop tôt pour en parler).

Il va de soi que la mise en ligne du portail ne marque pas la conclusion du projet : un répertoire est une œuvre située à la frontière des savoirs, fondée sur l'identification de ce que l'on sait, de ce que l'on doit encore découvrir, ou simplement de ce que l'on n'a pas encore enregistré. C'est pourquoi notre répertoire numérique sera ouvert à des intégrations, des corrections et des améliorations futures. Une sorte de chantier collaboratif dont le principe fondateur est, depuis l'origine, la mise en partage des connaissances.

Traduit de l'italien par Pierre Musitelli 